Revue des patrimoines

23 | 2014

Le patrimoine dans la Grande Guerre

\title{
Des expérimentations légitimées par le traumatisme : Paul Léon à Saint-Remi de Reims
}

\section{Camille Bidaud}

\section{(2) OpenEdition}

\section{Journals}

Édition électronique

URL : http://journals.openedition.org/insitu/10974

DOI : $10.4000 /$ insitu. 10974

ISSN : 1630-7305

Éditeur

Ministère de la Culture

\section{Référence électronique}

Camille Bidaud, « Des expérimentations légitimées par le traumatisme : Paul Léon à Saint-Remi de Reims », In Situ [En ligne], 23 | 2014, mis en ligne le 03 mars 2014, consulté le 15 novembre 2019. URL : http://journals.openedition.org/insitu/10974 ; DOI : 10.4000/insitu.10974

Ce document a été généré automatiquement le 15 novembre 2019.

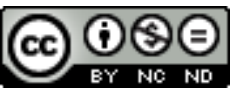

In Situ Revues des patrimoines est mis à disposition selon les termes de la licence Creative Commons Attribution - Pas d'Utilisation Commerciale - Pas de Modification 4.0 International. 


\title{
Des expérimentations légitimées par le traumatisme : Paul Léon à Saint-Remi de Reims
}

\author{
Camille Bidaud
}

\section{Introduction}

1 Le service d'Architecture du sous-secrétariat aux Beaux-Arts, dirigé par Paul Léon, s'efforça pendant la guerre de protéger les monuments historiques et de préparer la reconstruction grâce à une nouvelle organisation administrative, des expositions et de nouveaux partenariats. Il s'agit ici de donner une vision globale de ces actions, en abordant plus particulièrement le cas de la ville de Reims.

2 Alors qu'à la veille de la Première Guerre mondiale, la doctrine officielle avait évolué jusqu'à renoncer à la restauration pour ne pratiquer plus que la stricte conservation des monuments, l'étude de la restauration de Saint-Remi de Reims nous permettra de montrer comment la doctrine a su s'adapter aux pratiques engendrées par les destructions de la guerre.

3 Les principales sources utilisées sont à la Médiathèque de l'Architecture et du Patrimoine qui conservent, outre les dossiers de tous les Monuments Historiques, les archives personnelles de Paul Léon. Sur la période et sur Paul Léon pourront aussi être consultés " 1913, genèse d'une loi sur les monuments historiques » paru fin 2013, et la biographie de Paul Léon par Françoise Bercé.

\section{Paul Léon}

4 Depuis 1907 et pendant la guerre, Paul Léon (1874-1962) est chef de la division d'Architecture au sous-secrétariat des Beaux-Arts. En raison de son action pendant la Grande Guerre, il est nommé directeur des Beaux-Arts en 1919, fonction qu'il occupe 
jusqu'en 1932 malgré les nombreux changements de gouvernement. Agrégé d'histoiregéographie, et bien que ce poste soit avant tout administratif, son goût personnel le porte vers les monuments historiques, dont il se fit l'historiographe. Membre de la commission supérieure des Monuments historiques pendant plus de cinquante ans, il achève sa carrière comme professeur d'histoire de l'art monumental au Collège de France de 1933 à 1944 (il est cependant suspendu à cause des lois raciales de 1941 à 1945). Au titre des responsabilités diverses qu'il a exercées à la direction des Beaux-Arts et de son rôle de commissaire adjoint des expositions universelles de 1925 et 1937, il fut décoré de la Grand-Croix de la Légion d'honneur et élu membre de l'Académie des Beaux-Arts dès 1922.

\section{L'action du service d'architecture du sous-secrétariat aux beaux-arts à Reims}

\section{Monuments historiques et propagande}

5 En août 1914, la guerre éclate : la Belgique puis le nord de la France sont rapidement envahis. Reims est occupé par l'Allemagne le 4 septembre. La bataille de la Marne, entre le 6 et le 12 septembre, provoque le repli des Allemands. Mais, alors que la guerre a tout juste commencé, les destructions sont déjà lourdes. Avant même d'entrer en France, les A llemands détruisent par les flammes la bibliothèque de Louvain. Mais c'est le bombardement de la cathédrale de Reims, le 19 septembre, qui, provoquant l'incendie de la charpente et transformant la cathédrale en une gigantesque ruine, marque le plus les $\mathrm{F}$ rançais.

6 Paul Léon décrit cette tragédie comme le résultat d'une opération programmée, participant de la stratégie militaire, dans son ouvrage La vie des monuments français, destruction restauration, publié en 1951 :

Guerre de destruction totale [...] Ce n'était plus la torche jetée dans le désordre de la bataille et l'excitation du pillage. Des corps d'incendiaires étaient constitués, de même que d'autres avaient pour fonctions administratives l'enlèvement systématique des mobiliers et des stocks. La destruction et le rapt, considérés jusqu'alors comme des maux inévitables de l'invasion, étaient organisés en services publics. [...] guerre de peuple à peuple visant l'épuisement de toutes les ressources nationales : déportation des habitants, destruction des produits du sol, destruction de l'histoire elle-même par l'incendie des monuments qui en demeuraient le symbole ${ }^{1}$.

7 La propagande française expose alors la barbarie de l'Allemagne et son choix, politique, de détruire un monument d'art et d'histoire servant d'hôpital (pour des soldats allemands!). L'objectif de la France est de convaincre les pays neutres de s'engager auprès d'elle dans la guerre puisque l'Allemagne viole la convention IV de La Haye du 18 octobre $1907^{2}$, qui traite des lois et coutumes de la guerre sur terre.

Les deux pays s'affrontent aussi par l'intermédiaire de leurs services des Beaux-Arts, se lançant dans une surenchère pour la meilleure protection des objets mobiliers, la meilleure restauration d'édifice. Paul Clemen, professeur d'histoire de l'art à l'université de Bonn et alors inspecteur des monuments artistiques pour la Belgique et les territoires français envahis, et Paul Léon, entre autres, polémiquent par articles interposés, vantant les doctrines et techniques de leurs administrations respectives. 
Paul Clemen critique la France sur son attitude durant les dernières décennies vis-à-vis de son patrimoine, invoquant le vandalisme révolutionnaire, la loi de séparation de l'Église et de l'État et les maigres moyens dédiés à la conservation et à la restauration des monuments historiques ${ }^{3}$. Dès la fin de la guerre, les Allemands publient en plusieurs langues, dont le français, un ouvrage justifiant encore leurs actions pendant le conflit, avançant que la cathédrale de Reims n'a été atteinte que par quelques tirs de sommation en septembre 1914, pour qu'elle cesse de servir de poste d'observation, et par un obus perdu en 1918, alors que la Révolution française a causé bien plus de dommages à elle seule que trois siècles de guerres ${ }^{4}$. L'État allemand montre sa bonne volonté en restaurant la collégiale de Saint-Quentin, sous le feu français, et d'autres églises françaises, considérant que le mouvement gothique appartient à une tradition culturelle commune.

Côté français, la propagande prend des formes diverses: il s'agit, tout d'abord, de montrer la barbarie allemande en dressant une liste des monuments détruits, tel le rapport d'Arsène Alexandre, ou les nombreux articles sur la cathédrale de Reims.

11 Mais on s'attache aussi à décrier l'art allemand, comme Émile Mâle qui affirme dans L'Art allemand et l'Art français du Moyen-Age (Paris, A. Colin, 1917) que l'art allemand n'est fondé que sur le pastiche, tant pour les périodes romanes que gothiques ${ }^{5}$. Quant à Paul Léon, en 1923, il insiste sur l'origine francilienne du mouvement gothique ${ }^{6}$.

12 Enfin, on cherche à confronter les questions doctrinales de restauration. En effet, les Allemands opéraient selon la restauration stylistique viollet-le-ducienne, qui était remise en cause en France depuis une trentaine d'années. Paul Léon y répond directement dans son ouvrage La Renaissance des Ruines en exposant les excès de cette pratique à travers deux simples photographies avant/après de restaurations allemandes en France :

À Thann, le remplacement des statues anciennes de la façade par une galerie à colonnettes de la plus burlesque invention, l'invraisemblable coloris de la toiture, la décoration peinte de la nef, permettent de juger si notre abstention n'est pas préférable à leur zèle ${ }^{7}$.

13 Les questions doctrinales étant au cœur des discours de Paul Léon pendant et après la guerre, il participe à la propagande anti-allemande en défendant la doctrine française à travers de nombreux articles et ouvrages: «Les monuments et la guerre », paru en 1916 dans la revue Les Arts, ou encore La Renaissance des Ruines, en 1918.

Il publie aussi en 1917 son ouvrage principal, écrit avant la guerre, Les Monuments historiques, Conservation, Restauration, une histoire du service des Monuments historiques et une description de l'évolution des doctrines et pratiques en cours, où il explique dans la préface :

Cette étude pose plus de problèmes qu'elle n'en résout et présente un recueil de faits plutôt qu'un corps de doctrines. Elle était achevée quand la guerre a porté sur notre sol les ravages d'un vandalisme dont l'organisation même constitue la pire forme de la barbarie. J'ai cru qu'il ne serait pas inutile d'évoquer les leçons du passé devant les ruines du présent ${ }^{8}$.

Les Allemands, s'ils reconnaissaient l'importance des écrits et l'érudition de Paul Léon, tournaient ces arguments à leur avantage pour prouver le vandalisme de l'État français`. 


\section{Protection des monuments historiques et actions menées pendant la guerre} la ligne de feu les biens mobiliers de l'État et la statuaire. Les vitraux des cathédrales et de nombreuses églises furent démontés et emportés. Paul Léon rendit souvent hommage à Jacques Simon, peintre-verrier, qui démonta de nuit, sans échafaudage mais accroché à des cordes, les vitraux de la cathédrale de Reims. Lors de la dernière offensive allemande de 1918, les dépôts furent déplacés plus à l'intérieur des terres. Dans la mesure du possible, les monuments furent protégés par des sacs de sable et des travaux d'urgence exécutés. pour évaluer les dommages de guerre et collecter les œuvres à placer dans les dépôts. Ses archives personnelles contiennent de nombreux rapports sur les problèmes de déplacement des biens protégés et témoignent des difficultés rencontrées auprès de certaines municipalités qui refusaient que l'administration récupère les trésors locaux, comme les tapisseries de l'archevêché de Reims, devenues propriétés de la Ville, dont le maire s'opposa au départ et qui furent détruites quelques jours plus tard dans un incendie.

Dès 1918, un hommage public à l'action des services de Paul Léon est rendu par l'Assemblée nationale, dont Albert Dalimier, précédemment sous-secrétaire d'État aux Beaux-Arts, se fait l'écho :

Je voudrais d'un mot affirmer que, dans le passé, des efforts d'organisation ont été faits sous la direction de M. Paul Léon, à qui l'on rendait tout à l'heure un hommage si mérité, non seulement pour la protection des monuments, sur le front, mais pour le retrait de toutes les œuvres d'art qui pouvaient être exposées à un danger quelconque et qui ont été ramenées à l'intérieur du pays ${ }^{10}$.

Paul Léon obtient du service des armées la création d'une section photographique qui constitue une documentation sur les biens meubles et immeubles et les opérations de guerre, ce qui permit, en 1918, d'avoir de nombreuses traces des monuments et des villes avant leur destruction. Il instaure également un service d'action artistique à l'étranger, destiné à la propagande extérieure de la France. Dans ses souvenirs, il en expose le principe :

Je mis également sur pied, un service d'action artistique à l'étranger. N'était-il pas nécessaire, pour répondre à la puissante propagande germanique, de montrer au monde la France sous son vrai visage ? Nulle part elle ne devait être absente, malgré ses pertes, ses blessures, les menaces qui pesaient sur elle. En 1916 l'Exposition de San Francisco vint en porter le témoignage ${ }^{11}$.

Ces deux services voulus par Paul Léon furent fondés au ministère de la Guerre en 1915 pour servir de base à la propagande française durant la guerre. En 1919, ils furent menacés de disparition mais Paul Léon réussit à les transformer en associations privées placées sous le patronage de l'État ${ }^{12}$.

21 La reconstruction fut envisagée très tôt, puisque la loi de finances de 1915, votée le 26 décembre 1914, envisage déjà les réparations des dommages de guerre. Des commissions locales doivent évaluer les dégâts, mais aucune disposition n'est prise spécifiquement pour les monuments historiques. Au sein de l'administration des BeauxArts, Paul Léon doit se battre pour que le service fasse partie des commissions et que son 
avis soit consulté pour les réparations d'édifices anciens, même non classés. Il finit par obtenir gain de cause et le service est représenté dans le comité interministériel pour la Reconstitution des régions dévastées, fondé par le décret du 18 mai $1916^{13}$. Par la suite, il réussit à imposer que des membres de la commission des Monuments historiques et du conseil des Bâtiments civils fassent partie des commissions de reconstruction et que l'on puisse demander l'avis de ces services même si les édifices concernés n'étaient pas de leur ressort car non protégés au titre des monuments historiques ${ }^{14}$.

Mais ses prérogatives de chef de la division de l'Architecture au sous-secrétariat des Beaux-Arts l'amènent également à travailler sur la reconstruction des régions envahies. Dès 1916, le gouvernement décide que la France devra être reconstruite à l'identique. Les usines, édifices publics, habitations seront replacés sur leurs sites, selon la clause de « remploi en identique similaire». Une circulaire relative à l'établissement d'un plan général d'alignement et dénivellement dans les communes atteintes par l'événement de guerre, le 27 septembre 1916, prépare la loi Cornudet du 14 avril 1919 qui oblige les villes de plus de 10000 habitants à se doter d'un plan d'aménagement, d'extension et d'embellissement pour

...assurer, dans la plus large mesure possible, la conservation des souvenirs historiques et archéologiques, le maintien du style d'architecture spécial à la région, le respect des paysages, des sites et des aspects pittoresques qui représentent une part importante du patrimoine artistique et moral de nos populations et que leur valeur d'art et de souvenir doit nous rendre particulièrement chers dans les circonstances actuelles ${ }^{15}$.

Différentes expositions pratiques sont organisées à Paris. En 1916, l'exposition de la « cité reconstituée » sur les modèles de baraquements variés en matériaux légers, économiques et faciles d'assemblage, a lieu aux Tuileries. En 1917, c'est une exposition sur les types régionaux des provinces envahies, selon l'idée que "c'est bien en effet le caractère même du pays que reflètent les divers types de l'habitat humain ${ }^{16}$ ".

Cette exposition est suivie d'un concours d'architecture pour des projets de maisons rurales, fermes et auberges, économiques, gardant les caractéristiques régionales et répondant aux nouveaux canons hygiéniques pour toutes les zones envahies afin de préparer la reconstruction ${ }^{17}$. Paul Léon publie alors La Renaissance des Ruines, ouvrage consacré aux actions menées par ses services et aux doctrines qui devront guider la reconstruction de l'architecture ordinaire comme de l'architecture monumentale.

Pour la protection de la ville de Reims, et particulièrement de la cathédrale, Paul Léon fait démobiliser en 1915 Max Sainsaulieu ${ }^{18}$, architecte ordinaire des Monuments historiques. Ce dernier établit des rapports quasi quotidiens sur les bombardements subis par les monuments classés, réalise de nombreuses photographies utilisées par le service et la propagande et a en charge l'évacuation des objets classés.

De même, Henri Deneux (1874-1969), architecte en chef des Monuments historiques chargé de Reims, est envoyé en mission pendant la guerre à travers la France pour dresser des relevés de charpentes des églises et cathédrales. Ce travail lui servit plus tard pour la reconstruction des charpentes de la cathédrale de Reims, ainsi que des églises Saint-Jacques et Saint-Remi. Il donna aussi, après la guerre, des cours à Chaillot et fit des maquettes pour le musée du Trocadéro. 


\section{Opérations après-guerre (Une implication locale)} est exemplaire.

Pour Paul Léon, les sociétés savantes de province étaient importantes dans la prise de conscience nationale de l'intérêt des monuments anciens. Ce rôle se retrouve dans la composition de la commission des Monuments historiques, puisque, juste après la guerre, le nombre d'archéologues, antiquaires et historiens de l'art est augmenté.

Il fait partie lui-même de quelques sociétés, comme la société des Amis du Vieux Reims, association fondée au début du XXe siècle par Hugues Kraft et Ernest Kalas. Pendant la guerre, Paul Léon fait obtenir à l'association le parrainage du président de la République et la reconnaissance d'utilité publique. C'est sans doute grâce à son entremise que le siège de cette société s'établit pavillon de Marsan, au Louvre, lorsque Reims est évacué.

Il travaille avec Kalas à sa série de plans de Reims ${ }^{19}$ et lui demande d'en dessiner pour la reconstruction de la ville, qu'il publie plus tard dans un article de la revue Les Arts ${ }^{20}$, et qui ont probablement illustré ses cours au Collège de France.

À la fin de la guerre, il charge la société d'une mission officielle :

J'ai l'honneur de vous informer que vous êtes chargés d'une mission, à titre gratuit, ayant pour objet de réunir tous renseignements et documents pouvant être utiles à la commission des Monuments historiques pour la conservation et la restauration des monuments de Reims, et de représenter la Société des Amis du Vieux Reims aux opérations de déblaiement des ruines de la ville ${ }^{21}$.

En effet, dans les archives de la société se trouvent des copies d'une liste de monuments et d'éléments d'architecture à protéger d'urgence établie en 1919 et portant la mention « copie envoyée à Paul Léon ». De plus, la société se réunit régulièrement pour étudier les plans de reconstruction, formuler ses observations sur les destructions en cours...

En 1920, Hugues Kraftt fut nommé membre de la commission des Monuments historiques sur proposition de Paul Léon. Il participa régulièrement aux réunions de la commission jusqu'à sa mort en 1933.

\section{Saint-Remi de Reims : une restauration à l'épreuve de la guerre}

Après les nombreuses restaurations du milieu du XIX ${ }^{\mathrm{e}}$ siècle considérées au début du XX siècle comme abusives, le service des Monuments historiques prône une plus stricte conservation des édifices. Mais les trop nombreuses destructions de la Première Guerre mondiale contraignent le service à conduire de nouvelles restaurations, et donc à adapter son discours doctrinal pour expliquer ce choix. Le cas de Saint-Remi de Reims sur ce sujet

Paul Léon évoque cette église dès 1922 dans son discours sur la reconstruction de la France aux cinq Académies. Il en parle aussi beaucoup lors du congrès archéologique de France de 1934, et lui consacre un cours au Collège de France en $1935^{22}$. Mais il est intéressant de noter que dans aucun de ses discours, il ne mentionne que seule la restauration de la nef est terminée : les travaux du chœur ne furent achevés qu'en 1958.

De plus, il explique que Saint-Remi fut restauré, dans les grands traits, de la même manière que la cathédrale de Reims (charpente en béton armé, remontage des murs en anastylose avec parfois des structures en béton). Le cas de la cathédrale de Reims est trop 
connu et particulier pour être étudié ici, mais il faut rappeler que d'une part, sa charge symbolique imposait une reconstruction à l'apparence identique pour l'opinion publique et d'autre part, le don Rockefeller posait comme l'une de ses conditions la reconstruction en béton armé de la charpente ${ }^{23}$.

Enfin, Saint-Remi présente un cas intéressant en soi pour l'histoire de la restauration du patrimoine, puisque sa structure du XIII ${ }^{e}$ siècle était instable. Au XIXe siècle, l'église avait été restaurée en supprimant la voûte en pierre pour la remplacer par une voûte en bois. Après les bombardements de 1914, la voûte en pierre fut réinventée.

\section{L'église Saint-Remi ${ }^{24}$}

Saint Remi fut enterré en 533 hors les murs de Reims, dans la petite chapelle SaintChristophe, située au milieu d'un ancien cimetière. Rapidement devenue un lieu de pèlerinage, la chapelle fut élevée au $\mathrm{VI}^{\mathrm{e}}$ siècle au statut de basilique et consacrée à saint Remi. Elle fut agrandie par l'archevêque Hincmar en 852.

Le rôle de cette basilique est important dans l'histoire de Reims et de France puisqu'elle conserve le tombeau de saint Remi et la Sainte Ampoule, utilisée jusqu'à la Révolution française pour le sacre des rois de France. Les rois faisaient un pèlerinage à l'église le lendemain de leur couronnement.

L'église fut reconstruite de 1005 à 1049 pour pallier les décrépitudes de la construction d'Hincmar. En 1005, l'abbé Airard entreprit un ouvrage bien trop grand pour les moyens du monastère et les capacités techniques de l'époque. Son successeur l'abbé Thierry fit détruire à partir de 1039 l'œuvre d'Airard pour simplifier et diminuer l'ampleur de la construction. À son décès, en 1045, l'église était pratiquement terminée. Hérimar lui succéda et poursuivit le chantier et la basilique fut consacrée en 1049. L'église d'Hincmar avait alors complètement disparu.

Le moine Anselme, présent à la cérémonie, a écrit l'histoire de l'église et de sa consécration solennelle par le pape Léon IX. Son texte sert de base à toutes les histoires de l'église parues depuis mais a été remis en cause à la fin du XIXe siècle. Les fouilles effectuées après la Première Guerre mondiale ont permis de confirmer la véracité du document d'Anselme ${ }^{25}$.

En 1170, l'abbé Pierre de Celle ${ }^{26}$ refit le chœur en style gothique et deux travées de la nef, ajouta un déambulatoire à l'abside, transforma le portail entre les deux vieilles tours. Afin d'harmoniser le nouveau chœur gothique et la nef à charpente visible, il fit surmonter cette dernière d'une voûte en pierre qui devait reposer sur les dosserets appliqués aux murs et piliers romans (fig. $\mathbf{n}^{\mathbf{0}} \mathbf{1}$ ). Le poids important de cette voûte d'ogives obligea en réalité à créer des arcs-boutants à l'extérieur. Mais ces arcs-boutants ne furent placés qu'au rythme d'une colonne sur deux, ne permettant pas réellement l'équilibre des poussées. Dans l'église, les murs trop faibles pour supporter la voûte en pierre furent constamment restaurés. Lors de la Révolution, les statues et les tombeaux furent brisés, puis l'église désaffectée servit de manège. 


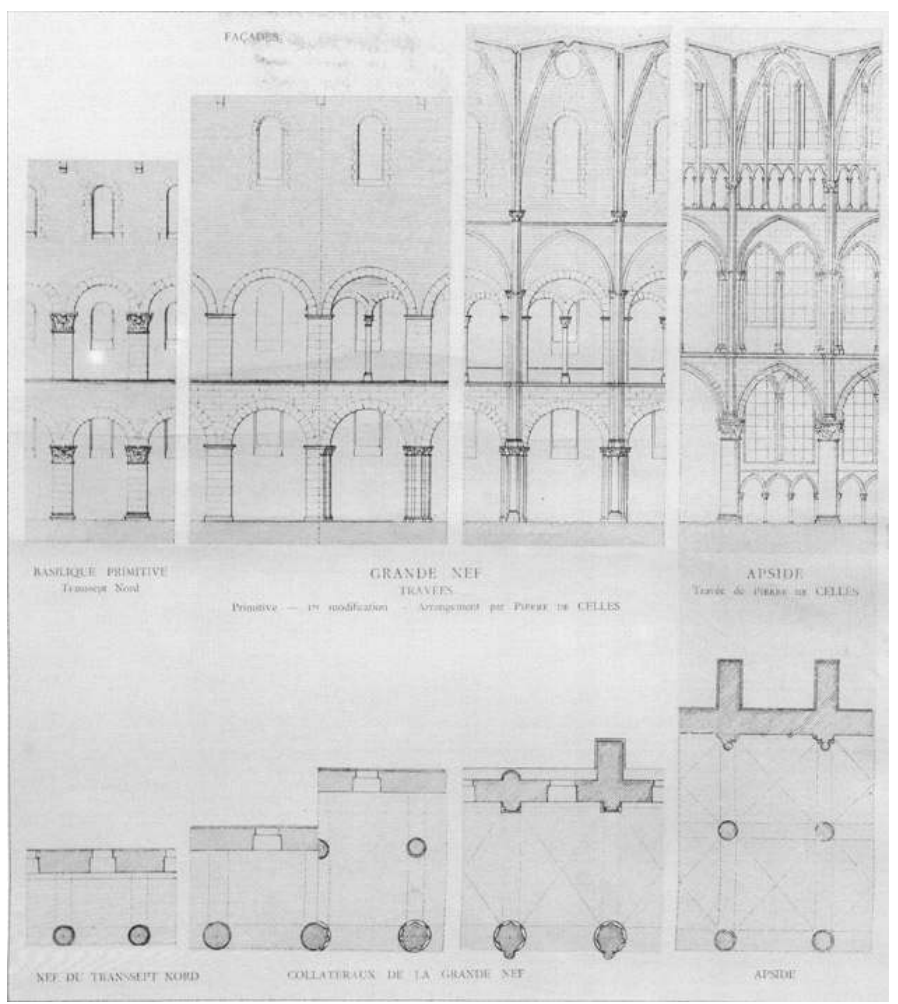

Plans et élévations de détail de la nef à différentes époques, par Alphonse Gosset (tiré de GOSSET, Alphonse. La basilique Saint-Remi à Reims, description, construction. Paris : Librairie centrale d'architecture, 1900).

En 1796, l'abbaye de Saint-Remi fut transformée en ambulance militaire puis en hôtelDieu. Lorsqu'elle fut rendue au culte, Narcisse Brunette, architecte de la ville et architecte diocésain à partir de 1837, relate que l'église était dans un état déplorable, n'ayant pas fait l'objet de travaux depuis longtemps, et que la nef était interdite au public dans sa totalité en raison de nombreux effondrements de la voûte ${ }^{27}$.

En 1825, à l'occasion du sacre de Charles X, l'architecte Jacques-Ignace Hittorf fit abattre la flèche de 1400 et la rosace du portail et étayer l'intérieur de l'église. Il explique que son travail devait être le début d'une grande restauration pour laquelle les fonds auraient été débloqués grâce à son rapport au conseil des Bâtiments civils ${ }^{28}$. Ces fonds n'arrivèrent malheureusement jamais.

Auguste Caristie, inspecteur général des Bâtiments, proposa de démolir les voûtes ainsi que les arcs-boutants, et de les remplacer par des voûtes en bois enduites de plâtre pour simuler la pierre et se raccorder au transept.

Nicolas Serrurier fut chargé en 1828 de la restauration proposée par Auguste Caristie, mais, suite aux problèmes techniques et aux dégradations plus importantes que prévu des murailles et $\mathrm{du}$ manque de flexibilité du système des adjudications de l'époque, les travaux n'avancèrent guère. Serrurier fut remplacé en 1836 par Durand qui proposa un nouveau devis, mais une partie des voûtes s'écroulèrent le $1^{\text {er }}$ septembre 1837. Il fut alors imaginé de supprimer toute la nef et de ne garder que le transept et le chœur. En août 1838, une commission d'experts conduits par Caristie conclut en faveur de la restauration de Saint-Remi, qui fut classée au titre des Monuments historiques en 1841. 

en partie le programme de Caristie et refit les voûtes d'ogives en bois et plâtre mais sans faux-joints imitant l'appareil de pierre de taille et il conserva les arcs-boutants. Il refit de plus le pignon du portail et une tour attenante. revient beaucoup sur les dégradations de Saint-Remi, et livre ensuite plusieurs pages sur sa restauration ${ }^{29}$. Mais il y évoque surtout l'économie que cette dernière représente par rapport à une reconstruction monumentale, sans évoquer la reconstruction de la voûte en bois. Narcisse Brunette ne fait qu'une allusion rapide au portail dont il dit avoir remplacé le couronnement du pignon sans avoir besoin de demander de nouveaux crédits grâce à d'autres économies sur le devis originel. Il est possible qu'il n'ait alors pas demandé l'autorisation de la commission des Monuments historiques.

Louis Demaison, en 1911, décrivait les travaux de Narcisse Brunette en ces termes :

Il fut réduit à la fâcheuse nécessité de démolir la voûte en pierre de la nef, dont la poussée était toujours menaçante, et de la remplacer par une voûte plus légère en bois et en plâtre, sorte de trompe-l'œil d'ailleurs assez bien réussi. On peut le regretter, mais on a du moins pu conserver intacts les arcades, les murs et les galeries de la nef. Le rétablissement de l'ancienne voûte de pierre n'aurait sans doute pu s'opérer qu'au prix d'une de ces reconstructions complètes, que l'on a trop souvent infligées à nos monuments historiques et qui leur enlève en partie leur valeur et leur caractère ${ }^{30}$.

Paul Léon prend pour exemple la restauration des voûtes dans Les monuments historiques, conservation, restauration ${ }^{31}$ pour montrer les mutilations du $\mathrm{XIX}^{\mathrm{e}}$ siècle. S'agissant du portail, il le classe parmi les fantaisies consécutives aux travaux de Viollet-le-Duc et à une méthodologie défaillante.

En 1906, l'architecte en chef des monuments historiques Charles Genuys (1852-1928) établit une terrasse en ciment armé au-dessus de la tribune, côté nord. Il s'agirait, d'après Paul Léon, d'une des premières applications du ciment armé aux toitures. C'est cette opération qui assura au mur nord du transept une meilleure tenue que le mur sud après la guerre. Il restaura en 1910 le pignon nord du transept.

L'église était alors considérée comme sauvée des vicissitudes du temps, prête à supporter de nouveaux siècles.

\section{La restauration : principes et campagnes}

52 En 1916, des bombardements détruisirent les chapelles absidiales, et le $1^{\mathrm{er}}$ aout 1918, un projectile mit le feu aux charpentes et aux voûtes en bois de la nef.

L'architecte désigné pour faire les travaux est Henri Deneux. Il avait été chargé d'une mission d'étude pendant la guerre sur les charpentes médiévales françaises, suite à la destruction de la charpente de la cathédrale de Reims. Il restaura en parallèle Saint-Remi, Saint-Jacques et la cathédrale.

Sans voûte ni berceau, les arcs-boutants de Saint-Remi ne contre-balançaient plus aucune poussée, ils exerçaient alors un effort de déversement vers l'intérieur, surtout du côté sud. Par manque de main-d'œuvre (la démobilisation n'eut lieu pour certains que dans l'été 1919) et de matériaux, le mur sud n'était pas étayé et s'effondra le 14 avril 1919 (fig. $\mathbf{n}^{\circ} 2$ ). Le déblaiement méthodique des ruines, travée par travée, grâce à l'intervention de prisonniers allemands, permit alors de comprendre les techniques de construction et de 
trancher des débats historiques sur les dates et formes de construction. Le 20 novembre 1920, la pointe du pignon du croisillon nord s'écroulait à l'intérieur de l'église en démolissant les voûtes avoisinantes.

Figure 2

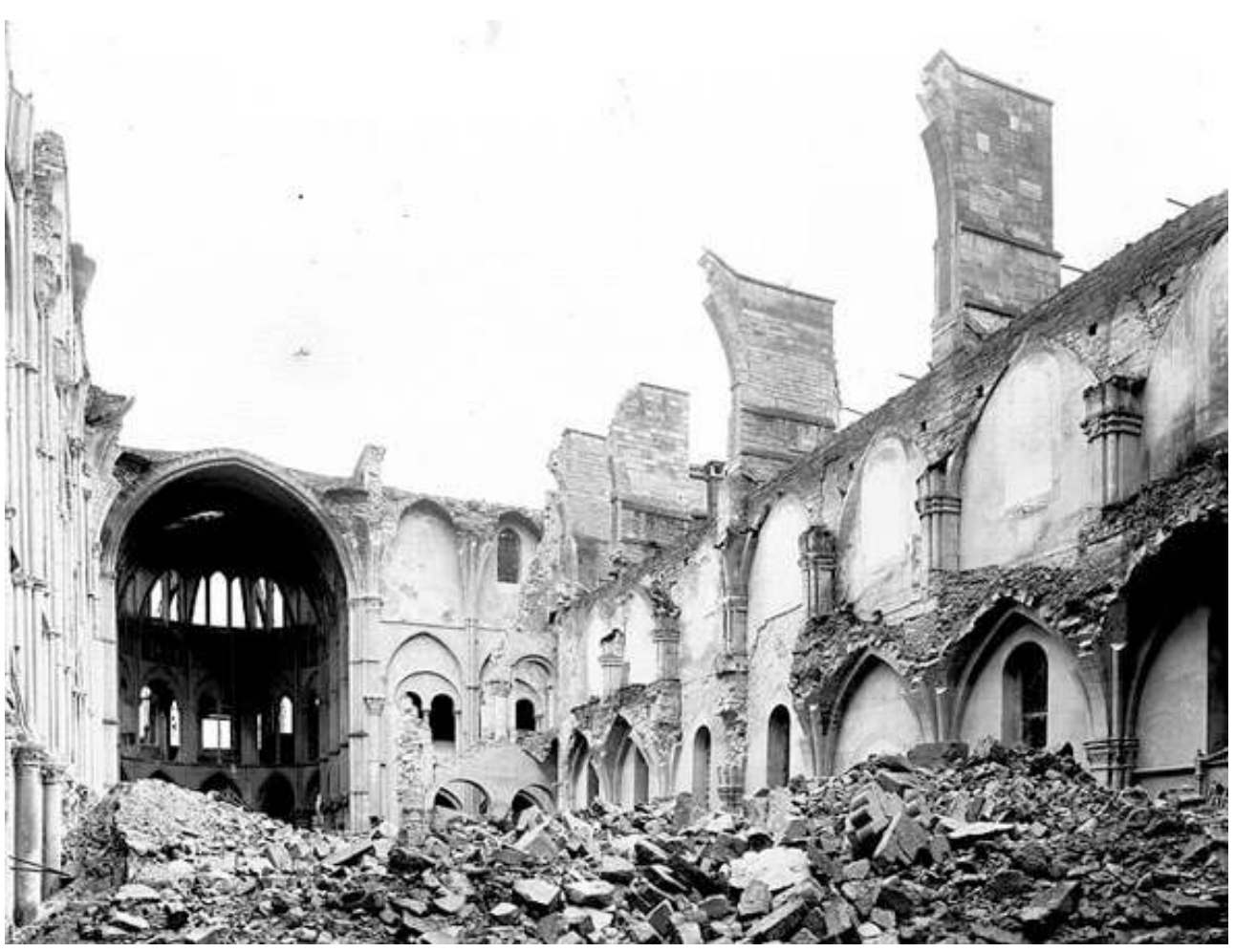

Ruines intérieures de l'Église Saint-Remi de Reims par Eugène Lefèvre-Pontalis, en 1919, base Mémoire nLP006293.

(c) Ministère de la Culture (France) - Médiathèque de l'architecture et du patrimoine - diffusion RMN.

Après le déblaiement, la première opération de restauration eut pour but de rendre une partie de l'édifice au culte, ce qui était demandé par le cardinal Luçon ${ }^{32}$. Il s'agissait de fermer le bas-côté nord, qui, protégé par sa terrasse en ciment armé, avait moins souffert que le reste de l'édifice. Le 23 mai 1920, une première messe fut célébrée dans cette petite partie.

La préoccupation première fut de rendre l'édifice au culte. Dans son ouvrage programme de la reconstruction La Renaissance des ruines, maisons, monuments, Paul Léon considère comme primordial le rétablissement du culte pour aider la population à retrouver un semblant de vie normale ${ }^{33}$.

En 1921 la reconstruction réelle commença. Le mur sud est tout d'abord remonté, en anastylose, avec les pierres retrouvées sur place, puis les arcs doubleaux de la nef et les arcs-boutants sont reconstruits ou réparés. Les pierres manquantes sont remplacées par des pierres de même qualité de couleur légèrement différente. Enfin, la voûte en pierre est réinventée. Paul Léon explique le choix de reconstruire la voûte en pierre :

Il était donc impossible d'obtenir un équilibre entre les arcs et la voûte. Au XIX ${ }^{\mathrm{e}}$ siècle, l'architecte Narcisse Brunette esquiva la difficulté en remplaçant les voûtes de pierres par des voûtes de bois. Dans les travaux d'après-guerre, adopter cette solution était un aveu d'impuissance. Il fallait voûter l'édifice comme il l'était au XII

${ }^{e}$ siècle, assurant sa solidité sans modifier son aspect ${ }^{34}$. 
58 prononcé en 1922, il revient sur les doctrines et leurs applications ${ }^{35}$ :

59 L'église étant aujourd'hui découverte, il importe de lui restituer ses voûtes primitives, mais leur poussée, désormais, se trouvera annulée par un cadre en ciment armé dissimulé dans le comble et relié aux tas de charge. Un tel mode de renforcement permet de conserver des édifices que leur délabrement aurait jadis condamnés ${ }^{36}$.

60 En pratique, les arcs doubleaux sont surmontés d'un «corset » en ciment armé qui les empêche de se déformer. Les corsets sont indépendants de la maçonnerie afin de pouvoir se dilater. Ils servent aussi à déplacer les points de poussée des arcs-boutants (fig. $\mathbf{n}^{\circ} \mathbf{3}$ ).

Figure 3

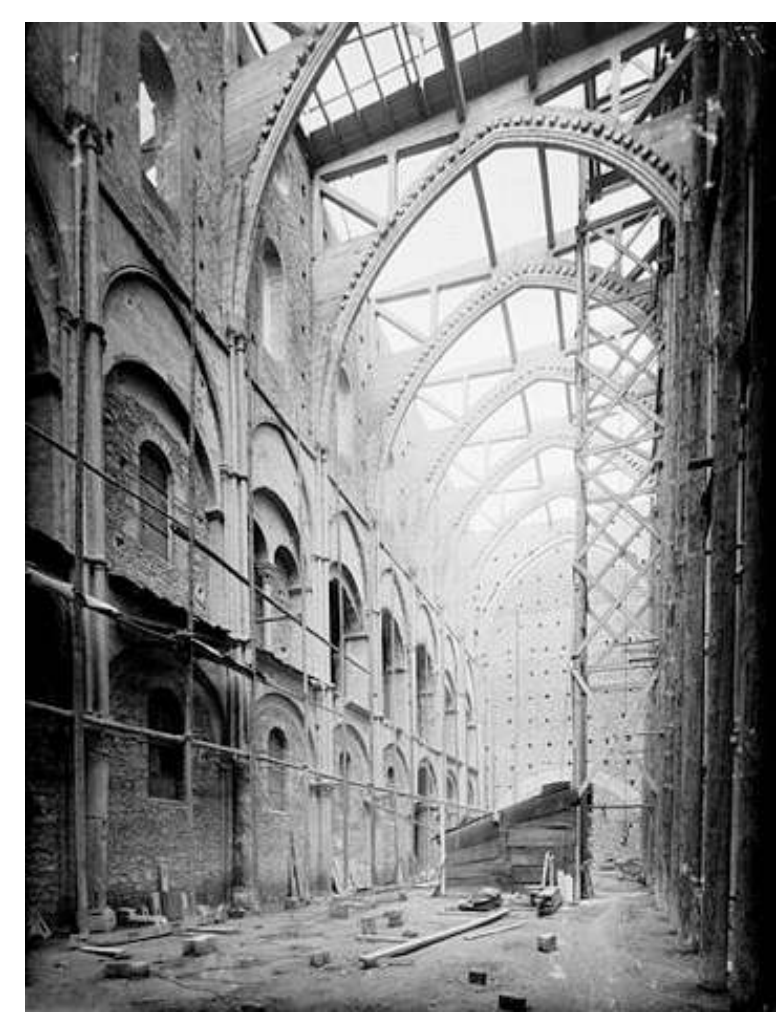

Les arcs doubleaux à l'Église Saint-Remi de Reims par Henri Heuzé, base Mémoire nMH0077962. (c) Ministère de la Culture (France) - Médiathèque de l'architecture et du patrimoine - diffusion RMN.

61 Entre 1927 et 1930 sont rétablies la charpente et la couverture. La charpente est réalisée, comme à la cathédrale, en éléments de ciment armé préfabriqués puis montés et assemblés avec des clavettes en bois suivant le type de la charpente à la Philibert Delorme. Le dessin de la charpente est original par rapport à la cathédrale car sa portée est plus grande d'un mètre et d'une plus grande longueur (fig. $\left.\mathbf{n}^{\circ} \mathbf{4}\right)$. 
Figure 4

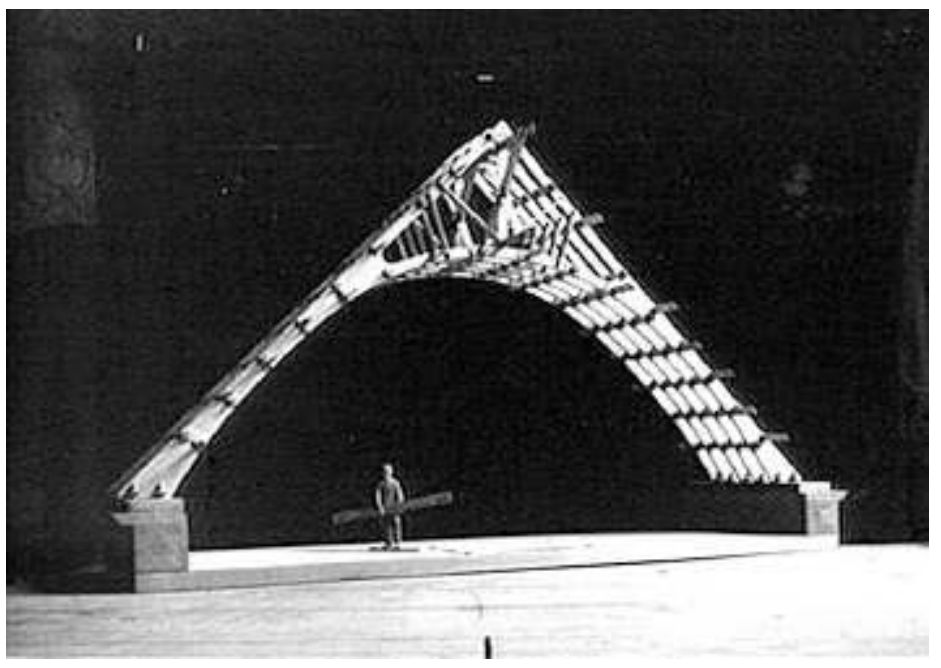

Maquette de la charpente de Saint-Remi de Reims par Henri Deneux, base Mémoire nDNX1713 V. (c) Ministère de la Culture (France) - Médiathèque de l'architecture et du patrimoine - diffusion RMN.

L'inauguration de la nef eut lieu le 4 octobre 1931, au début des neuvaines de saint Remi. La nef était alors séparée du transept par un mur en maçonnerie la protégeant du chantier qui continuait dans les absides et les transepts. La restauration fut ralentie par la réduction du budget des Monuments historiques à partir de 1934, puis interrompue pendant la Deuxième Guerre et jusqu'en 1950, faute de moyens. L'inauguration de la basilique entière n'eut lieu que le 12 octobre 1958.

\section{Une doctrine de restauration remise en question}

63 Henri Deneux a présenté dans les détails sa restauration aux membres de la société des Amis du Vieux Reims, réunis pour visiter l'église le 29 septembre 1931, soit quelques jours avant l'inauguration de la nef :

On ne pouvait à cause de leur intérêt archéologique, modifier la forme, ni le nombre, de ces arcs-boutants, qui sont, peut-être, avec ceux de Notre-Dame de Châlons, les plus anciens connus.

En présence de ces difficultés, et pour respecter entièrement l'aspect intérieur et extérieur de l'édifice, il fallait trouver une solution qui neutralisât la poussée de ces voûtes, sans tirants en fer placés, à l'intérieur, au niveau de la naissance des voûtes, et sans arcs-boutants nouveaux, à l'extérieur.

[...] Grâce au matériau nouveau employé, les difficultés énoncées plus haut furent vaincues, et la nef a pu reprendre son aspect ancien, avec sa voûte primitive en pierre $^{37}$.

$64 \mathrm{Au}$ nom de "l'intérêt archéologique » les arcs boutants sont laissés à l'identique, par « respect » pour l'aspect intérieur et extérieur ancien, les voûtes sont recréées telles qu'elles ont existé avant 1820. Mais ces voûtes, qui n'ont historiquement jamais été stables, le deviennent en 1931 par l'action, cachée et invisible, du béton armé. La valeur documentaire du monument, son intérêt pédagogique sur la statique médiévale sont donc perdus pour ceux qui n'ont pas connaissance des secrets des combles.

Paul Léon promut pendant sa carrière la conservation plutôt que la restauration, faisant siennes les idées de Ruskin (1819-1900). On considère alors que la doctrine de Viollet-le- 
Duc laisse trop de place à l'arbitraire, ce qui représente un changement de doctrine pour la France. À la conférence d'Athènes de 1931, Léon distingue trois périodes doctrinales en France vis-à-vis des monuments depuis la Révolution française :

«Une période empirique, celle des destructions et des palliatifs; une période doctrinale, celle des grandes restaurations; une période expérimentale, celle de la conservation ${ }^{38}$ ».

Les travaux à Saint-Remi de Jacques-Ignace Hittorf et de Narcisse Brunette appartiennent à la période empirique. La période doctrinale est celle des restaurations de Viollet-le-Duc et de ses disciples. Paul Léon considéra toute sa carrière, avant comme après-guerre, être dans la période expérimentale de la conservation. Il expliqua les raisons du passage de la seconde à la troisième période plus précisément dans son allocution à la conférence :

Depuis la fin du dernier siècle les architectes renonçant à reconstruire les édifices tels qu'ils ont dû exister, se bornent à les maintenir tels que nous les a légués le passé. La restauration fait place à la conservation. Ce changement a eu des causes très diverses. Tout d'abord l'extension du classement, [...] la loi de Séparation des Églises et de l'État [...] notre éclectisme ne proscrit plus aucune époque. [...] À l'extension des classements dans l'espace, correspond leur extension dans le temps. [...]

De nombreuses sociétés provinciales sont nées; elles multiplient leurs publications et leurs congrès. L'influence des archéologues s'est exercée sur l'éducation des architectes. [...] Les artistes, les critiques d'art n'ont cessé de protester contre les restaurations abusives, contre les copies d'œuvres d'art contraires au principe même de l'art. [...] «Le traitement du passé par le présent meurtrier, écrit Rodin, amène un deuil irréparable». La presse a joué un grand rôle [...]. Le public se passionne aujourd'hui pour ces questions. Le tourisme l'a rapproché des monuments et l'intéresse à leur vie.

Sous ces diverses influences, les méthodes administratives ont évolué. Depuis une vingtaine d'années un service d'entretien a été constitué, qui remet entre les mains des architectes locaux les crédits indispensables à la conservation des édifices. Leur prompte et efficace intervention permet souvent, à peu de frais, d'éviter d'onéreux travaux ${ }^{39}$.

67 Mais la ligne officielle de ces grands discours sur la conservation est en contradiction avec la reconstruction intégrale de Saint-Remi et de nombreux autres monuments. Pour Paul Léon, ces restaurations sont dues à l'immensité du désastre de la guerre. La théorie suivie était alors de ralentir les destructions du temps qui passe (la fameuse "béquille " de John Ruskin), mais les bombardements ne peuvent pas entrer dans la même catégorie comme il le justifie au congrès archéologique de France de 1934:

Fallait-il les laisser à l'état de ruines, pour témoigner devant l'histoire? Dans beaucoup de cas, c'était impossible ; [...] la cathédrale de Reims, sanctuaire de notre histoire, pouvait-elle être condamnée ? [...] Comme après la Révolution, il fallait arracher à la destruction totale des monuments en péril. Une profonde conscience, une méthode minutieuse, l'emploi des techniques modernes ont guidé ces restaurations... ${ }^{40}$

Paul Léon exposa plus précisément la méthode retenue dans la suite de son discours à Athènes :

Pour être de plus en plus rare et limitée dans ses effets, la restauration n'en est pas moins, dans certains cas, indispensable. La réédification n'est sans doute qu'un pisaller, mais elle est le seul moyen de conserver une forme et de faire vivre une pensée. Du moins doit-elle être entreprise avec un infini scrupule, une religieuse soumission à l'art d'une époque qui n'est plus. L'origine des pierres neuves, leur appareil, leur taille, leurs joints, doivent être toujours l'objet d'une surveillance attentive. [...]. La perfection des procédés actuels a fait ses preuves au cours des 
restaurations entreprises dans les régions dévastées qui utilisent les ressources de la technique moderne tout en conservant intact l'esprit même du Moyen Âge ${ }^{41}$. commencé très discrètement à être utilisé dans les monuments historiques au début du siècle. Les principaux essais sont réalisés avant-guerre, sur proposition d'Anatole de Baudot, qui fait décréter à la commission que le béton a un intérêt structurel indéniable, mais que l'esthétique n'étant pas satisfaisante, il ne doit pas être visible. Après la guerre, il est très utilisé dans les parties invisibles, toitures, charpentes, injectés dans les murs, en fondations...

70 Au Moyen Âge, toute la structure est visible, c'est elle qui dessine l'espace. Faire tenir structurellement l'édifice par du béton armé que l'on cache est contradictoire avec «l'esprit intact» du monument que l'on veut sauvegarder. Outre le fait que la valeur pédagogique est perdue par le principe de structure cachée, la valeur documentaire pourrait alors aussi être remise en cause.

71 Le 4 octobre 1931, Paul Léon prouve son implication dans cette restauration par sa présence à la cérémonie en tant que représentant du gouvernement :

Nos travaux n'ont rien à craindre de la rude épreuve des siècles. [...] Les constructions successives ne devaient pas résister aux fureurs de vandalisme suivis d'une longue période de négligent abandon. Il y a cent ans, les moyens dont disposaient les architectes n'ont permis que des solutions trop timides ou trop brutales : reconstruction de la façade, substitution de voûtes en bois à la charge des voûtes en pierre. La technique d'aujourd'hui se joue de pareils problèmes. C'est un singulier paradoxe que l'architecture moderne, rompant nettement par l'universel emploi des matériaux moulés ou plaqués avec l'antique construction par assise et refouillement ait si puissamment aidé à la conservation de nos monuments anciens auxquels elle est dans son principe, radicalement étrangère et qu'elle est, dans l'avenir, destinée à remplacer ${ }^{42}$.

Il est rare dans les écrits et discours de Paul Léon qu'il parle de l'architecture moderne. En effet, il parle volontiers de la conservation, de la restauration, de reconstructions traditionnelles mais rarement de constructions contemporaines. Mais il est conscient et ouvert aux possibilités qu'apporte le béton armé pour la restauration. Cette phrase sera d'ailleurs intégralement reprise deux semaines après l'inauguration à Athènes.

Parmi les conclusions de la conférence d'Athènes est entériné officiellement l'usage du béton pour les restaurations structurelles dans des parties invisibles :

[Les experts] approuvent l'emploi judicieux de toutes les ressources de la technique moderne et plus spécialement du ciment armé.

Ils spécifient que ces moyens confortatifs doivent être dissimulés sauf impossibilité, afin de ne pas altérer l'aspect et le caractère de l'édifice à restaurer ${ }^{43}$.

74 On peut remarquer l'importance des délégations françaises et italiennes à cette conférence. Après les grands débats durant la guerre entre Allemands et Français, la restauration stylistique est mise à mal au cours de la conférence, où elle n'est défendue par aucun participant.

75 La doctrine édictée par les conclusions de la conférence correspond à peu près à ce que prône Paul Léon au fil de ses discours: stricte conservation autant que faire se peut, importance de l'entretien régulier, protection des fouilles, restaurations rares et strictement encadrées, privilégiant l'anastylose, emploi du béton armé dans les parties invisibles, et prédominance du droit de la collectivité sur le droit privé... 

acceptée par les Français (en particulier par Pierre Paquet qui, dans la continuité de Mérimée, déclare que le but recherché est que l'on ne puisse percevoir la trace d'une restauration ${ }^{44}$. À Saint-Remi par exemple, Henri Deneux utilise de nouvelles pierres aux tons extrêmement proches des anciennes pierres réutilisées. Mais, contrairement aux conseils de Mérimée, elles ne sont pas patinées, ce qui les rend légèrement plus visibles.

Le choix de la reconstitution intégrale des édifices est éminemment politique, mais semble satisfaire autant les populations que les archéologues, pourtant généralement hostiles aux restaurations importantes. Seuls quelques artistes auraient préféré garder les ruines comme témoignage permanent de la férocité allemande. Mais les villes d'Arras ou de Reims n'auraient alors plus eu de centre historique. Et Paul Léon, conscient de l'écart entre la doctrine officielle de la conservation et la pratique de la restauration, conclut ses discours, tant à Athènes qu'au congrès archéologique, par cette réflexion :

Quoi qu'il en soit, au lendemain comme à la veille de la guerre, notre époque demeurera sans doute plus favorable aux travaux de stricte conservation qu'à ceux de grande restauration. Elle continuera de marquer pour les monuments anciens une époque de survie plutôt que de rajeunissement, plus soucieuse de les prolonger que de les ressusciter, et par là même, en dépit de l'aphorisme de Michelet, plus conforme à la Vérité et plus fidèle à l'Histoire ${ }^{45}$.

\section{NOTES}

1. - LÉON, Paul. La vie des monuments français, destruction, restauration. Paris : A. et J. Picard, 1951, p. 489.

2. - HARLAUT, Yann. La cathédrale de Reims, du 4 septembre 1914 au 10 juillet 1938. Idéologies, controverses et pragmatisme. Thèse d'histoire. Reims : université de Reims Champagne-Ardenne, 2006, p. 64 et suiv.

3. - CLEMEN, Paul. "Notre protection des monuments des arts en temps de guerre». Correspondance historique et archéologique, 1915. Traduction et commentaire par Louis Dimier d'un article originellement paru le 19 janvier 1915 dans le Zentralblatt der Bauverwaltung.

4. - CLEMEN, Paul. Berichte über den Zustand der Kunstdenkmäler auf den verschiedenen Kriegsschauplätzen und über die deutschen und österreichischen Massnahmen zu ihrer Erhaltung, Rettung, Erforschung. 1: Die Westfront [Protection de l'art pendant la guerre, rapports sur l'état des monuments d'art sur les différents théâtres de guerre et sur les mesures prises par les Allemands et les Autrichiens pour leur conservation, leur sauvetage, leur étude. Vol 1: Le front de l'Ouest]. Leipzig: E. A. Seemann, 1919, p. 139 et 113.

5. - HARLAUT, Yann, op. cit., p. 152.

6. - LÉON, Paul. L'art gothique et les grandes cathédrales: notice historique et archéologique. Encyclopédie des styles. Paris : Ducher, [1920].

7. - LÉON, Paul. «Les monuments et la guerre ». Les Arts, avril 1916, n¹54, p. 2-10 et LÉON, Paul. La Guerre et l'architecture : la renaissance des ruines ; maisons, monuments. Paris : H. Laurens, 1918.

8. - LÉON, Paul. Les Monuments Historiques, conservation, restauration. Paris: H. Laurens, 1917, incipit. 
9. - GRAUTOFF, Otto. «La conservation des monuments, au jugement de l'étranger ». Dans CLEMEN, Paul, op. cit., p. 113.

10. - Extrait des débats de la Chambre du 12 mars 1918, Journal officiel du 13 mars 1918, p. 885, cité par GRAUTOFF, Otto, op.cit.

11. - LÉON, Paul. Du Palais-Royal au Palais-Bourbon, souvenirs. Paris : A. Michel, 1947, p. 176. Une cloche partiellement fondue et certaines statues à moitié détruites par les bombardements de la cathédrale de Reims sont envoyées à l'exposition pour marquer les esprits américains.

12. - Paul Léon insiste beaucoup dans ses souvenirs sur ces services qu'il aurait fait créer et perdurer. Des recherches restent à faire, en particulier dans les archives du ministère de la Défense, pour voir quelle fut son implication réelle.

13. - AUDUC, Arlette. "Paul Léon, le service des Monuments historiques et la reconstruction, enjeux et cadre institutionnel ». Dans BULLOCK, Nicolas et VERPOES, Luc. Living with History, 1914-1964 : Rebuilding Europe after the first and second World Wars, and the Role of Heritage Preservation. Leuven : Leuven University Press, 2011, p. 73.

14. - Art. 4 du décret du 26 mai 1919, ibid.

15. - Extrait de la circulaire relative à l'établissement d'un plan général d'alignement et de nivellement dans les communes atteintes par les événements de guerre du 27 sept. 1916, tiré de LÉON, Paul. La renaissance des ruines, maisons, monuments. Paris : H. Laurens, 1918, p. 44.

16. - LÉON, Paul. «L'architecture régionale dans les provinces envahies ». Les Arts, $1917, n^{\circ} 157$, p. 12.

17. - LÉON, Paul. La renaissance des ruines, maisons, monuments. Paris : H. Laurens, 1918.

18. - Arrêté du 15 janvier 1915, arch. dép. de la Marne, 4 T 14.

19. - Une série des plans de Kalas, donnés à Paul Léon, a été retrouvée par Jean-Jacques Valette de la SAVR au musée d'Orsay, ce dépôt faisant probablement suite à la confiscation de la bibliothèque personnelle de Paul Léon sous l'Occupation.

20. - LÉON, Paul. « La reconstruction de Reims ». Les Arts, 1919, n¹72.

21. - Tiré d'une lettre de Paul Léon à Hugues Krafft (président de la société) datée du 11 mars 1919, archives de la SAVR, hôtel Le Vergeur, Reims.

22. - Carton 080/47/5 des archives de Paul Léon, Médiathèque de l'Architecture et du Patrimoine, cours daté d'après l'annuaire du Collège de France.

23. - Sur la restauration de la cathédrale de Reims et sa charge symbolique voir la thèse Yann Harlaut, op. cit.

24. - Références historiques tirées de BOUXIN, Marc. "L'Abbaye Royale de St Remi de Reims ». Dossiers d'archéologie, octobre 1993, n¹86, p.60-73, et DEMAISON, Louis. "Église Saint-Remi ». Congrès archéologique de France. LXXVIII ${ }^{\mathrm{e}}$ session tenue à Reims en 1911. Paris : A. Picard, Caen : H. Delesques, 1912, p. 57-106, qui fut publié à part : L'église Saint-Remi de Reims. Caen : H. Delesques, 1913 ; de CROUVEZIER, G. Saint-Remi de Reims. Reims : L. Michaud, 1957 et « Saint-Remi de Reims ». Bulletin Monumental, 1937, p. 91-100.

25. - Sur ce débat, voir : DEMAISON, Louis. La restauration de l'église..., op. cit. p. 92 ; ANSELME. Itinerarium Leonis Papae. Dès 1881 dans les travaux de l'académie de Reims, Louis Demaison défend la véracité du récit d'Anselme, à l'encontre de Viollet-Leduc (sic) et de ce qui est dit au congrès archéologique de France de 1875 à Châlons par Leblan (Congrès archéologique de France. XLII ${ }^{\mathrm{e}}$ session, séances générales tenues à Châlons-sur-Marne en 1875. Paris: Derache, 1876, p. 234); voir: DEMAISON, Louis. "Date de l'église de Saint-Remi de Reims ». Travaux de l'Académie nationale de Reims, 1881, vol. LXXI, p. 298-308.

26. - Sur les travaux de Pierre de Celle à Saint-Remi voir: PRACHE, Anne. Saint Remi de Reims, l'œuvre de Pierre de Celle et sa place dans l'architecture gothique. Bibliothèque de la société française d'archéologie. Genève : Droz, 1978.

27. - BRUNETTE, Narcisse. Souvenirs archéologiques et notes relatives à la ville de Reims. Meaux: Destouches, 1885, p. 35-36. 
28. - HITTORFF, Jacques-Ignace. « Rapport fait par M. Hittorff, au nom de la commission chargée d'examiner le projet de restauration de l'église abbatiale de Saint-Remi de Reims, par M. Durand, architecte, membre correspondant ». Annales de la société libre des Beaux-Arts, 1837.

29. - BRUNETTE, Narcisse. Souvenirs archéologiques... op. cit., p. 82-90.

30. - DEMAISON, Louis. L'église Saint-Remi de Reims. Caen : H. Delesques, 1913, p. 9-10.

31. - LÉON, Paul. Les monuments historiques, conservation, restauration. Paris: H. Laurens, 1917, p. 247. Il cite en référence l'article de Louis Demaison dans le Congrès Archéologique de France. Le chapitre de cette citation est repris quasiment sans changement par Paul Léon dans La vie des monuments français..., op. cit., p. 362.

32. - Voir HARLAUT, Yann. La cathédrale de Reims..., op. cit., dans le $\mathrm{V}^{\mathrm{e}}$ chapitre, la partie « un mythe associé : le cardinal Luçon », p. 116-124.

33. - LÉON, Paul. La renaissance des ruines..., op. cit., p. 68.

34. - LÉON, Paul. «Les principes de la conservation des monuments historiques, évolution des doctrines ». Congrès archéologique de France. Paris : A. Picard, 1936, p. 17-52.

35. - LÉON, Paul. La restauration des monuments après la guerre, communication lue dans la séance annuelle des cinq académies le 25 octobre 1922.

36. - LÉON, Paul. La restauration des monuments après la guerre, op.cit., p. 14-15.

37. - DENEUX, Henri. «La Restauration de la Basilique Saint-Remi, conférence de M. Henri Deneux ». Annuaire-bulletin de la société des amis du vieux Reims, 1931-1935, p. 22-35, p. 31.

38. - LÉON, Paul. «La restauration des monuments en France». Dans La conservation des monuments d'art et d'histoire. Paris : Institut de Coopération intellectuelle, 1933, p. 17-18.

39. - LÉON, Paul, ibid.., p. 56-57.

40. - LÉON, Paul. « Les principes de la conservation des monuments historiques »..., op. cit., p. 46.

41. - LÉON, Paul. « La restauration des monuments en France... », op. cit., p. 58-59.

42. - Extrait du discours prononcé par Paul Léon lors du déjeuner officiel suivant l'inauguration de Saint-Remi.

43. - Extrait des conclusions de la conférence d'Athènes, La conservation des monuments d'art... op. cit.

44. - PAQUET, Pierre. "Technique de la restauration des monuments historiques». Congrès archéologique de France. XCVII ${ }^{\mathrm{e}}$ session tenue à Paris en 1934. Paris: A. Picard, 1936, tome 1, p. 414-415.

45. - LÉON, Paul. «Les principes de la conservation des monuments historiques »..., op. cit., p. 51-52.

\section{RÉSUMÉS}

Ce texte étudie le rôle, pendant la Première Guerre mondiale, du service d'Architecture du soussecrétariat aux Beaux-Arts et de son directeur Paul Léon dans la protection et la reconstruction des monuments historiques des territoires proches de la ligne de front. L'étude porte principalement sur la ville de Reims. La doctrine des Monuments historiques, alors attachée avant tout, et depuis plusieurs décennies, à la conservation, est contrainte d'évoluer en raison des nombreuses opérations de restauration auxquels le service est confronté après guerre, comme nous le verrons avec le cas de Saint-Remi de Reims. Pendant cette période, Paul Léon utilise ces études et travaux à des fins de propagande. 
This article is a study of the architectural services at the French Sous-Secrétariat des Beaux-Arts, headed by Paul Léon, who has considerable importance in the protection and reconstruction of historic monuments in territories close to the front during the First World War. The article concentrates in particular on the city of Rheims. The official doctrine of the Historic Monuments administration, hitherto entirely devote to conservation, was forced to evolve on account of the numerous restorations immediately after the war, as we shall see with the case of Saint-Remi of Rheims. During the war and after it, Paul Léon used his studies and restoration work as propaganda tools.

\section{INDEX}

Mots-clés : Paul Léon, Monuments historiques, Saint-Remi de Reims, restauration, conservation, propagande, reconstruction, Service d'architecture, protection, Première Guerre Mondiale, doctrine, pratiques

\section{AUTEUR}

\section{CAMILLE BIDAUD}

Doctorante en architecture (ED VTT, laboratoire IPRAUS - UMR AUSser) camille@bidaud.net 